\title{
Need for a proactive and structured approach to risk analysis when designing phase I trials
}

\author{
Cornelis A Van den Bogert PhD candidate ${ }^{1}$, Adam F Cohen chief executive officer ${ }^{2}$ \\ ${ }^{1}$ Utrecht Institute for Pharmaceutical Sciences, Division of Pharmacoepidemiology and Clinical Pharmacology, University of Utrecht, Netherlands; \\ ${ }^{2}$ Centre for Human Drug Research, 2333 CL Leiden, Netherlands
}

Good news from Emanuel and colleagues ${ }^{1}$ - apparently, phase I clinical drug trials are safe. However, given the very low frequencies of serious adverse events, retrospective associations of trial characteristics with adverse events are of no use for predicting safety problems in future trials. None of these associations would have helped prevent the cytokine storm caused by TGN1412. This drug's risk originated from the molecule's pharmacology, which could have been identified and dealt with upfront. We therefore underscore the importance of a proactive (upfront) analysis of future trial risks.

A structural framework for such a risk analysis was reported in $2006 .{ }^{2}$ The building blocks relate to basic principles of pharmacology and use information from previous ex vivo and in vivo research. ${ }^{2}$ Assuming that the quality of the experimental compound is stable and volunteers are healthy, pharmacology is the main source of safety problems and the central area of investigation in phase I trials. So, upfront identification of what is already known about the pharmacology of a new compound and what knowledge the trial should add ought to be the essential objective of any rationally designed phase I trial. This facilitates a structured analysis of the risks of each of these aspects of the trial.
We agree that transparency is a big problem. ${ }^{3}$ All empirical data on the compound should be available, otherwise meaningful risk analysis is impossible.

In summary, a proactive and structured approach to risk analysis is needed. The successful reintroduction of TGN1412 (TAB08) in the clinic illustrates how good management of preclinical data and study design can change a dangerous molecule into a promising new drug. ${ }^{4}$ Retrospective analysis of adverse events may identify risks from the past but is unlikely to help when dealing with future unknowns.

Competing interests: None declared.

Full response at: www.bmj.com/content/350/bmj.h3271/rr-0.

1 Emanuel EJ, Bedarida G, Macci K, et al. Quantifying the risks of non-oncology phase I research in healthy volunteers: meta-analysis of phase I studies. BMJ 2015;350:h3271. (26 June.)

2 Kenter MJ, Cohen AF. Establishing risk of human experimentation with drugs: lessons from TGN1412. Lancet 2006;368:1387-91.

Kimmelman J. The secret realm of phase I trials in healthy volunteers. $B M J$ 2015;350:h3444. (26 June.)

4 Tabares P, Berr S, Romer PS, et al. Human regulatory T cells are selectively activated by low-dose application of the CD28 superagonist TGN1412/TAB08. Eur J Immuno I 2014:44:1225-36. 